\title{
Libertad como fin educativo que transforma la práctica de un aula llanera ${ }^{1}$
}

\author{
FREEDOM AS AN EDUCATIONAL PRACTICE IN ORDER TO TRANSFORM A CLASSROOM \\ PLAINS \\ A LIBERDADE COMO UMA PRÁTICA EDUCATIVA, A FIM DE TRANSFORMAR UMA SALA DE \\ AULA PLANÍCIES
}

\author{
Diana Yohana Gamboa Ladino* / mfed_dgamboa648@pedagogica.edu.co \\ Yohanna Milena Nieto Gamboa** / mfed_ynieto936@pedagogica.edu.co
}

\section{Resumen}

La educación señala un reto de formación comprometido con el fortalecimiento de las cualidades humanas frente a las expectativas de cada ser y las necesidades sociales. Tal compromiso se refleja en las acciones y prácticas que se asumen como una oportunidad para mejorar las capacidades de los seres humanos. De tal inspiración surge la necesidad de transformar la práctica educativa en un aula de primero de primaria en la ciudad Villavicencio, en la que se articulan compromisos, fines y acciones para construir experiencias de libertad desde la cotidianidad escolar.

\section{Summary}

Education notes challenging training committed to strengthening human qualities versus expectations of every being and social needs. This commitment is reflected in the actions and practices that are assumed as an opportunity to enhance the capabilities of human beings. From this inspiration comes the need to transform educational practice in a first grade classroom in the city Villavicencio, where commitments, goals and actions are articulated to build experiences freedom from school every day.

\section{Resumo}

Notas Educação desafiando formação empenhada em reforçar as qualidades humanas contra expectativas de cada ser e as necessidades sociais. Esse compromisso se reflete nas ações e práticas que são assumidos como uma oportunidade para melhorar as capacidades dos seres humanos. A partir desta inspiração vem da necessidade de transformar a prática educativa em uma sala de aula de primeiro grau na cidade de Villavicencio, onde compromissos, metas e ações são articuladas para construir experiências liberdade da escola todos os dias.

\section{Palabras clave}

Experiencias de libertad, práctica educativa, Investigación Acción Educativa.

Keywords

Experiences of freedom, educational practice, Educational Action Research.

\section{Palavras-chave}

Experiências de liberdade, prática educativa, Ação Educativa Research.

\section{Fecha de recepción: 12 de junio de 2014 / Fecha de aceptación: 31 de octubre de 2014}

1 El presente artículo surge de la investigación "Experiencias de libertad: un desafío para la práctica educativa", realizada en el marco de la Maestría en Desarrollo Educativo y Social, a través del convenio Fundación Centro Internacional de Educación y Desarrollo, Cinde, y la Universidad Pedagógica Nacional (2012-2013).

* Licenciada en Pedagogía Infantil, Universidad Distrital Francisco José de Caldas. Maestrante en Desarrollo Educativo y Social. Docente de básica primaria, Institución Educativa Las Palmas, Sede Brasilia, Villavicencio (Meta).

** Licenciada en Educación Básica con énfasis en Ciencias Sociales, Universidad Pedagógica Nacional. Becaria Maestría Desarrollo Educativo y Social. 


\section{Introducción}

En el presente escrito se presentan reflexiones en torno al sentido que adquiere investigar las propias prácticas, a partir de la experiencia de una maestra que trabaja con niños de primero de primaria en la institución Educativa Las Palmas (IELPA), Municipio de Villavicencio (Meta). La investigación se orientó a partir de la pregunta: ¿Cómo se construyen experiencias de libertad en la práctica educativa de una docente de primero primaria en el municipio de Villavicencio?

Para encontrar respuestas a dicho interrogante, se consideró como objetivo principal comprender la manera cómo se construyen esas experiencias de libertad en la práctica educativa de la docente. Inicialmente se identificaron acciones de esa práctica que se relacionan con experiencias de libertad; luego, se reflexionó con el propósito de reconstruir la comprensión sobre el quehacer docente desde esta perspectiva. Además, se continuó con una problematización del sentido de la práctica educativa como ejercicio autorreflexivo, con compromiso social, ético y político.

De acuerdo con las intenciones conjuntas que llevaron a desarrollar la investigación sobre la propia práctica educativa, se determinó que el enfoque metodológico más adecuado era la Investigación Acción Educativa (IAE), en cuatro momentos claves: decisión para cambiar y actuar; revisión y fundamentación teórica, que se fue construyendo en torno a las prácticas educativas y al concepto de libertad; reflexión sobre el quehacer docente; y por último, la fase de reconfiguración investigativa que transformó la práctica educativa.

Este recorrido condujo a plantear como tesis principal que la convivencia escolar es conflictiva cuando se impone una única manera de vivir, trabajar con otros y participar del colectivo, pero cuando la práctica educativa planea, diseña y crea experiencias de libertad, la actitud de aprendizaje se enfoca en una educación para la vida digna, donde se valora la construcción del "juntos" para vivir, trabajar, para encargarse de sí mismo, para dar lo mejor de sí y para habitar propositivamente con el mundo.

\section{La escuela: el escenario de construcción de experiencias de libertad y prácticas educativas esperanzadoras}

Cuando la convivencia escolar se tornó más y más conflictiva en el grupo de primero de primaria, hubo que hacer una pausa para entender por qué se imponían los gritos, las acusaciones, las quejas y, en especial, la dependencia de los estudiantes hacia la profesora encargada. Así, se reconoció que inicialmente la práctica educativa era heterónoma; entonces surgió la pregunta por la posibilidad de propiciar y comprender alternativas de autonomía que contribuyera a una educación en y para la libertad.

Al indagar sobre la importancia de la libertad en la historia de la humanidad, se encontró que la esclavitud y la opresión han sido los causantes de grandes sometimientos e intensas luchas, tal como lo sugiere Pattersson (1991), quien recuerda que desde el pensamiento de los griegos del siglo V y IV a. C., se ha defendido la libertad como principio en las dimensiones personal, cívica y soberana. Según Pattersson (1991, p. 32), para alguna parte de la sociedad griega, la esclavitud significó identidad, seguridad y pertenencia cultural, pero la defensa de la libertad pasó de ser una demanda individual, a convertirse en un principio colectivo, gracias a la conciencia femenina. Sin embargo, soltarse de un yugo como la esclavitud o la servidumbre, puede ser entendido como una vivencia de libertad, en tanto esto sea producto de la decisión y tenacidad de quienes así la viven. Savater (2004) sintetiza que la libertad es poder tomar decisiones para sí mismo y ser consciente de esto:

“La libertad es poder decir 'sí' o 'no'; lo hago o no lo hago, digan lo que digan mis jefes o los demás; esto me conviene y lo quiero, aquello no me conviene y por tanto no lo quiero. Libertad es decidir, pero también, no lo olvides, darte cuenta de que estás decidiendo" (pp. 50-51).

Se espera que los adultos, niños y jóvenes tengan la capacidad de decir "sí" o "no" a eso que el mundo pone de presente. Pero si desde la infancia, en la familia y en la escuela, siempre hay alguien que representa la autoridad y decide absolutamente todo para quienes 
están a su cargo, es muy difícil que tal sujeto, en edad adulta, tome decisiones por y para sí mismo.

Aquí se estima que la libertad no es un estado de aislamiento individual, sino una conquista existencial y colectiva para deshacerse de los límites que impiden vivir dignamente; además, la libertad personal, aunque necesaria, no es suficiente para que la humanidad se resista a aceptar una vez más la esclavitud o la servidumbre. Así que escuchar al propio yo que reclama y manifestarse, no es suficiente, se hace necesario construirla con otros y eso sobrepasa los límites de la libertad personal.

Es ahí donde se considera que la educación puede proponer alternativas de convivencia que enriquezcan la dignidad humana desde lo que se hace en el aula de clase, exaltando como principio ético la libertad en el proceso de formación, porque se cree que la educación puede y debe afirmar cualidades, valores y elementos para que cada integrante de la práctica reconozca su protagonismo en la construcción de la propia biografía y del colectivo en el cual participa.

De esta manera se comparte con Sen (1999), que: "El aumento de la libertad mejora la capacidad de los individuos para ayudarse a sí mismos, así como para influir en el mundo, y estos temas son fundamentales para el proceso de desarrollo" (p. 33). En consecuencia, se propone que el desafío en la libertad cívica, aquí entendida como libertad colectiva, consiste en superar las limitaciones y privaciones que condicionan el desarrollo, para que la comunidad incremente la gobernabilidad en y para sus propias prácticas.

Esa idea de libertad entendida como capacidad, desafía al ser humano a una tarea más grande de ser, porque ya no se trata de lograr unos objetivos personales o deseos inmediatos; en la libertad colectiva se aspira a lograr unos referentes organizativos y participativos que le permitan al resto de las personas identificarse con una causa y defenderla, ahí radica la importancia de que los fines se traduzcan en acciones $y$, por ende, en prácticas; entonces, si la libertad es colectiva, la humanidad valora su profundidad material y espiritual.
El politólogo Berlín (2004) hace énfasis en la relación de la libertad con: "las carencias que impiden a los seres humanos poder disponer de eso que se desea ser o hacer y que depende de las propias capacidades para alcanzarlo" (p. 221). Aquí la libertad entonces es entendida como la superación de alguna condición, circunstancia, individuo o grupo que impide que otros sean o hagan conforme a lo que se valora como vida digna, porque se habla de una libertad que reconoce el derecho adquirido por y para la naturaleza humana, no solo para el individuo.

En suma, la relación entre libertad y educación se articula a partir de la conciencia de vivir con otros, es decir, en la convivencia cotidiana que suscita aprendizajes de reconocimiento y fortalecimiento de capacidades para vivir con otros. Ante ese desafío, se avanzó con la siguiente ruta metodológica para lograr los aprendizajes que se describen a continuación.

\section{Ruta metodológica para investigar las propias prácticas}

La ruta metodológica llevó a la configuración y reconfiguración de las categorías centrales y subcategorías. Dados los descubrimientos en la realidad educativa, y la paulatina conciencia del proceso transformador para las investigadores en su quehacer docente, implicó un mayor compromiso en asumir las apuestas metodológicas que sugiere el enfoque de Lewin, citado por Kemmis y McTaggart (1988, p. 17), quien describió la Investigación Acción Educativa como un proceso de peldaños en espiral, cada uno de los cuales se compone de planificación, acción, observación y reflexión- evaluación.

De tal proceso se consolidaron como categorías centrales las experiencias de libertad y la práctica educativa. A la luz de la cotidianidad escolar, se agruparon en las subcategorías para poder comprenderlas, estas son: aprender a vivir y trabajar juntos, a ser dueño de sí mismo, a dar lo mejor de sí, y a habitar con el mundo. 
Aprendizajes en el aula

A continuación, se presentan los aprendizajes logrados en la práctica educativa con el grupo de primero de primaria en la Institución Educativa Las Palmas, sede Brasilia.

\section{Aprender a vivir y trabajar juntos}

Hacerse cargo de la propia biografía se encuentra como el ejercicio más coherente con aprender a vivir, a tomar decisiones, a trabajar con otros para alcanzar sueños o crear ideas, para reconocer en el otro que la existencia es un milagro de la vida y, por tanto, es valiosa. Puig (2010) es uno de los pedagogos que consideran que el propósito de la educación es aprender a vivir; en sus palabras, esto es: "una tarea educativa que querría liberar a los individuos de sus limitaciones, ayudándoles a establecer vínculos basados en la apertura y la comprensión de los demás y en el compromiso en proyectos a realizar en común" (p. 65).

En la misma línea, Bárcena (2005) reafirma la necesidad de aprender a vivir, pero bajo una circunstancia especial: la incertidumbre.

"Lo que necesitamos es aprender a vivir, actuar y pensar bajo la incertidumbre que tanto tememos. En este sentido, en la medida en que ya no parece posible el proyecto de coordinación entre el esfuerzo por racionalizar el mundo y el de preparar seres racionales adecuados para habitarlo; y en la medida en que -tras los horrores científicamente asistidos del siglo XX, que han puesto en jaque la creencia de que la ciencia podría humanizarnos- se ha desvanecido la esperanza de un dominio racional del hábitat social, la única alternativa quizá sea hacer que la educación consista en una verdadera preparación para la vida, es decir, el cultivo de la capacidad para vivir cotidianamente en paz con la ambigüedad, la incertidumbre, la adversidad de puntos de vista, los desacuerdos y la inexistencia de autoridades infalibles y fiables" (p. 37).

Bárcena expone una reflexión educativa con conciencia histórica que invita a una formación para la capacidad de vivir en paz aún a pesar de estar inmerso en la adversidad de la vida misma. La adversidad por la que el grupo Primero Uno pasaba, era la falta de respeto en el trato mutuo. Por lo que inicialmente se desmontó la pretensión de certezas para lanzarse al riesgo de los descubrimientos en convivencia, fue así como surgió la pregunta por la capacidad autónoma de los niños, justo frente a la frustración de darse cuenta que la indicación de no gritar, no golpear a otro o no interrumpir a la profesora, se había quedado en el discurso.

\section{7 de marzo 2013}

"En estos días, tanto mis estudiantes como yo no hemos hecho otra cosa que borrar los valores de nuestra convivencia, que nos propusimos construir desde un comienzo, nos olvidamos de no gritar, no nos escuchamos, no nos respetamos y no nos reconocemos como grupo. Pienso nuevamente que hemos perdido cualquier señal de libertad, al menos el camino lo hemos extraviado".

La profesora relata su angustia al no encontrar coherencia con los compromisos adquiridos en los primeros días de clase, cuando se estableció el pacto de aula1. Pero construir normas con niños de 6 a 8 años es un desafío de largo aliento, porque ellos apenas están aprendiendo a desarrollar su capacidad reflexiva para considerar al otro, a los otros. Es claro que una cosa es aprender a escuchar y otra a obedecer, a mantener la boca cerrada, porque no se trata de mantener el silencio incólume, sino de aprender a desarrollar habilidades sociales para vivir y trabajar con los demás. Escuchar es ponerse en disposición de atender al otro, mirarle a los ojos, concentrarse en lo que dice e interactuar.

\section{Aprender a ser dueño de sí mismo}

En el contexto escolar, aprender a ser dueño de sí mismo significa encargarse de uno de manera responsable. Se trata de un ejercicio de poder ser libre. Durante el seguimiento a la convivencia del grupo, se comprendió que lo que subyace a las experiencias de libertad transita entre el poder individual y el colectivo, para hacerse cargo del trabajo que implica educarse con otros y aprovechar situaciones cotidianas para convertirlas en experiencias, lo cual convocó la autodeterminación del grupo, el autoconocimiento de la profesora, la reinterpretación de la autoridad, la confianza en las capacidades propias, voluntad, formación del carácter, iniciativa y, sobre todo, responsabilidad ética, política y moral.

1 El Pacto de Aula hace referencia a un acuerdo de convivencia sugerido por la institución educativa. 
Las experiencias de libertad, desde la perspectiva educativa, y encaminadas al aprendizaje de ser dueño de sí, provocaron un desacomodamiento en quienes escriben. Luego de algunos días de profunda reflexión se identificó que para ser dueño de sí es necesario fortalecer la confianza y templar el carácter. Así, se asumió una de las expectativas en el primer año de escolaridad, aprender a leer y escribir.

\section{3 de mayo de 2013}

"Aprender a leer puede ser igual que como aprendieron a montar bicicleta, seguramente se cayeron y se dieron en la cabeza, se rasparon las rodillas, pero así, golpe tras golpe, se levantaron y continuaron, y hoy no se arrepienten de nada, es más, gozan calle arriba y calle abajo manejando la bici".

Cuando los niños son conscientes de que los adultos confían en ellos, logran entender que están creciendo y que están ganando independencia. Es como el tránsito del triciclo a la bicicleta de dos ruedas: se conquista la alegría de la autodeterminación y la autonomía. Ser dueño de sí mismo es también ser dueño del cuerpo, poder decidir cuándo ir al baño o no, es básico como experiencia de libertad. Durante las conversaciones con la profesora de este grupo, se comprendió que el desorden empieza cuando el poder y el control de cada acto se centra en ella, es por esto que prefiere hacer ver que cada uno tiene injerencia sobre su propio ser.

\section{2 de febrero de 2013}

"Sobre los permisos, creo también que si los estudiantes van al baño de la manera más natural, así como lo hacen seguramente en su casa, de manera tranquila, no tiene por qué existir ningún problema. Si los chicos desde pequeños hacen esto y lo naturalizan, no tendrán por qué creer que la ida al baño es una opción para escapar de las aburridas clases. Estoy convencida de que esta cuestión de no pedir permiso para ir al baño sirve como pretexto para construir autonomía y control sobre nuestro cuerpo y nuestras acciones".

En la desmedida entrega de autonomía de los estudiantes a la profesora, también se ha perdido bastante control sobre el propio cuerpo, y esto vulnera la libertad más elemental de los seres humanos. Consciente de esto, ella ha aprendido que la libertad no solo está en un grito de sublevación, sino que está en las prácticas mismas, en lo que se hace para permitir o impedir que otro pueda ser.

\section{Aprender a dar lo mejor de sí}

Ante los conflictos, situación cotidiana en la escuela, se busca culpables para castigar e inocentes para defender o victimizar. Educar en libertad alienta a que los directamente implicados en un conflicto busquen alternativas de solución que sean justas para las partes, y esto requiere de un proceso de negociación, de descentrarse, pensar y considerar al otro. Sucede con mucha frecuencia que en los grupos de clase hay uno o dos estudiantes que constantemente chocan con el resto del grupo y paulatinamente se convierten en los responsables de cualquier problema que surja.

\section{7 de febrero}

"Juan le hizo zancadilla a un niño de segundo, y como iba corriendo cayó de manera brusca sobre una niña pequeña de 1-2 que salió muy lastimada. Inmediatamente otra profesora me dijo que debía abrirle proceso a este estudiante, llevar registro en el observador y así demostrar que esa clase de estudiantes no nos los tenemos que aguantar en el colegio. En fin, yo le respondí que lo iba a hacer, sin embargo, iba a escribir en el observador que era un incidente en medio de un juego. Todo quedó así, hablé con Juan, quién se reconoció culpable. Le dije que no creía que fuera culpable, que sí había cometido un error o una falta al jugar de esa manera, pero que su intención no era lastimar a la niña (Él asintió con la cabeza). También le dije que era necesario que se disculpara y así lo hizo".

Es para los profesores un reto alentar el amor propio de cada estudiante, pero en especial hacer equilibrio entre la solución de los problemas y su bienestar. No obstante, durante las discusiones, quejas y reclamos, no es sencillo tener la lucidez necesaria, por esto se considera tan importante reflexionar sobre el carácter educativo que tiene una sanción.

Los conflictos en el medio escolar requieren de una intervención inmediata, por eso se hace necesario 
que el estudiantado se fortalezca con sus pares y padres de familia, en cuanto a la toma de decisiones en conjunto y avanzar hacia horizontes comunes. En este sentido, toda la comunidad educativa debe participar activamente de estos debates $y$, principalmente, son los profesores los encargados de diseñar y aplicar las prácticas que consideran formativas para sus estudiantes; de esta forma se construye la cultura moral de las instituciones educativas, tal como lo afirma Puig (2012): "Los educadores construyen la cultura moral de un centro educativo, al idear, organizar y poner en marcha un conjunto de prácticas formativas apropiadas para la correcta formación de su alumnado" (p. 88).

\section{Aprender a habitar con el mundo}

Este aprendizaje invita a entender el desafío ético y moral de vivir en un país privilegiado en elementos que permiten y favorecen la vida humana. Se trata entonces de aprender con los estudiantes que se puede ser mejores huéspedes de la naturaleza, de entender que estamos de paso, y por eso, además de ser responsables de sí mismos, es necesario cuidar al perro, al gato, al agua, a todo lo que manifieste el milagro de la vida.

Actualmente la institución educativa se encuentra en una casa alquilada temporalmente, mientras se termina de construir su propio colegio que está a algunas cuadras. Esta situación ha generado incomodidad porque la temperatura aumenta en las horas de la tarde, se genera ansiedad por tomar líquido fresco y el colegio no cuenta con agua potable. La región cuenta con una importante riqueza hídrica (ríos, quebradas, riachuelos), entonces la profesora procura promover el valor del líquido en medio de la sed a través del compromiso de sus estudiantes para evitar que las fuentes de agua sean contaminadas. Probablemente esta dificultad sea superada cuando se trasladen a su propia sede, pero mientras tanto, esto es lo que sucede:

\section{9 de marzo 2013}

"El colegio se abastece de un pequeño aljibe que se encuentra en el patio, justo al lado de los baños, este pozo casi siempre está seco y la institución se queda sin una gota de agua, ni siquiera para lavarse las manos. Hay 2 tanques elevados para el agua, sin embargo no son suficientes para 425 estudiantes de bachillerato y primaria.
Y se agrava la situación porque este aljibe no tiene agua, además, la calidad del agua es aún más grave, alguna vez levanté la tapa del pozo, vi dentro papeles de la basura que se alcanzan a filtrar cuando llueve, es decir que las aguas Iluvias van a parar al pozo, y con ellas todo lo que pueda circular por el patío, también he visto un sapo inflado que flotaba sobre el agua, y de las Ilaves sale el agua con residuos, no sé si es musgo o qué cosa verde es, y el agua huele mal".

En esta parte de las prácticas, Freire (2011, p. 35) fue de nuevo quien alentó y renovó la fuerza para hacerse cargo y continuar recordando que eso que se aprecia en la realidad es producto de las acciones humanas y, por tanto, su recreación puede estar en manos de la misma especie. Ya se ha visto que transformar la realidad es para este grupo un reto diario, y por eso ese miércoles se decidió volver a "humanizar" el parque con actos que estaban en manos de los propios estudiantes.

\section{5 de junio de 2013}

“Después del descanso, nos fuimos para el parque a colorear unas imágenes para construir un mural del día mundial del medio ambiente. Llevaban maletas y todo lo necesario para trabajar, les dije: antes de salir, observarán ¿cómo estaban las calles? si limpias o ¿había basura?".

Los niños resolvieron recoger los papeles, uno de ellos se ofreció a comprar una bolsa de basura para guardarlos ahí. Para la profesora esto representó un símbolo de compromiso. Ellos disfrutaron de salir porque en el parque hay más ventilación que en el salón, pero además contribuyeron a que el parque estuviera más limpio, fueron conscientes de eso, jugaron y regresaron al colegio. Hasta aquí se asume que la construcción de experiencias de libertad no es necesariamente el desarrollo de acciones extraordinarias por su organización, sino de experiencias valiosas por el valor ético que significan. Las libertades personales que se alcanzaron, como poder decidir cuándo ir al baño, dieron vida a esa idea de libertad colectiva que procuró buscar el bien para ellos como niños, estudiantes, y para la profesora, quien ha aprendido a transformarse entre crayolas y experiencias de libertad. 


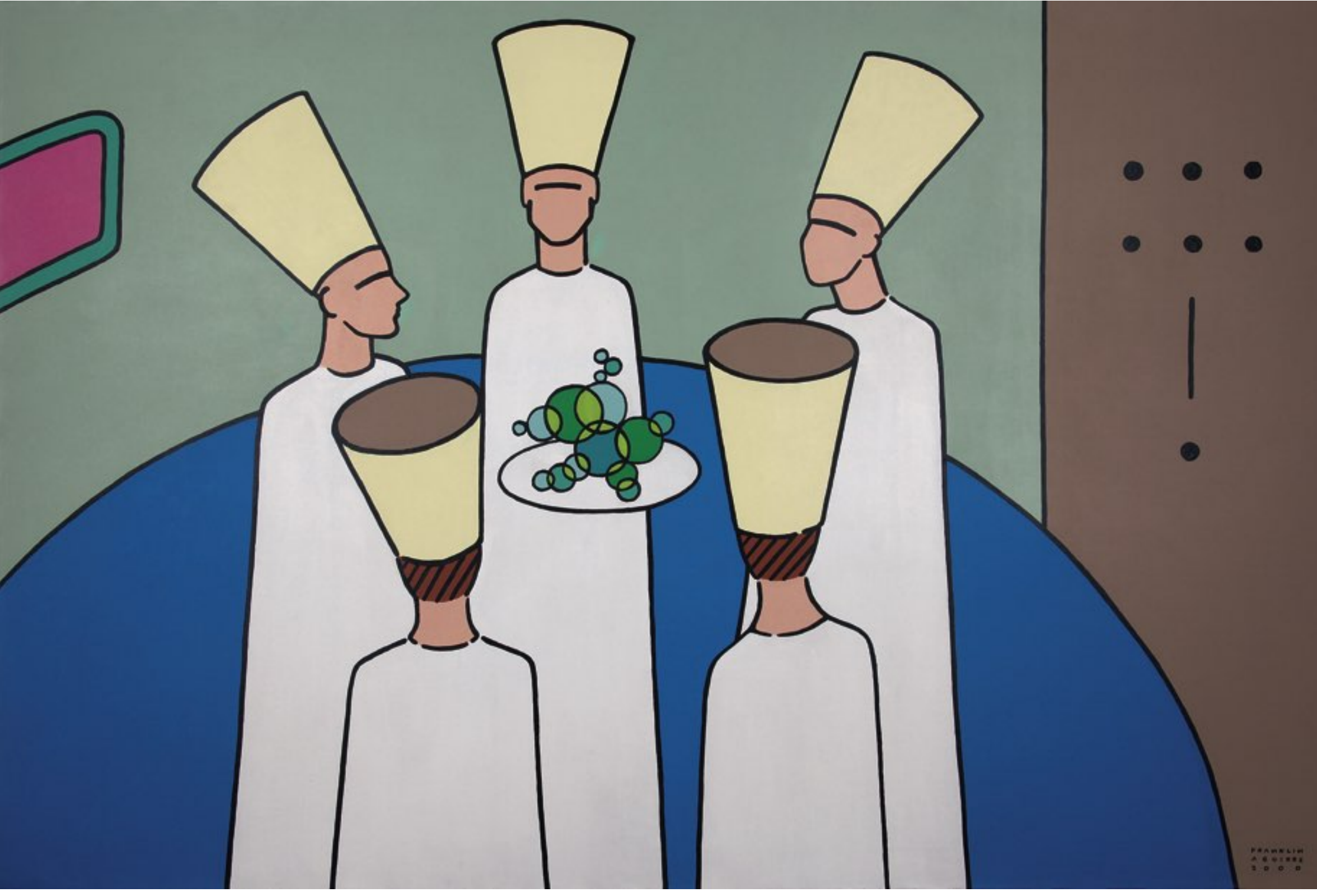

Franklin Aguirre ॥ Título: Octubre ॥ Técnica: Acrílico sobre lienzo ॥ Tamaño: 100 X 70 cm 


\section{Aprendizajes al investigar sobre las propias} prácticas

Al analizar las narraciones y el material audiovisual obtenido, se logró identificar tres etapas de la transformación a la práctica: 1) Las limitaciones y resistencias institucionales, que promovían la heteronomía entre los escolares, incrementaron la falta de seguridad y responsabilidad entre los niños; 2) Las transformaciones percibidas por la profesora y los estudiantes en la disminución de agresiones, ofensas y mejoramiento en la actitud hacia el trabajo colectivo; y 3 ) Los aprendizajes percibidos permitieron concluir que una educación para la vida, necesariamente debe y puede apoyar capacidades para poder ser y hacer, también para dejar ser y hacer; entonces es ahí donde se considera oportuno suscitar el tránsito entre la libertad personal a la cívica, porque ésta amplia la confianza, la seguridad, y se traduce en propuestas éticamente responsables y políticamente comprometidas.

Esta investigación provocó un movimiento de "sacar" lo mejor de las autoras en el campo investigativo y pedagógico, de lo cual se desprende la conciencia del papel que ocupó la IAE como hilo conductor para comprender las experiencias de libertad durante la práctica educativa que orienta la profesora encargada del grupo primero de primaria.

En suma, ser libre se refleja en la capacidad de reconocer qué es lo que limita la libertad y trabajar con el colectivo para superarlo; ahí radica la potencia de que la libertad sea asumida como uno de los fines en educación. En cuanto a las capacidades que desarrolla un docente investigador, éstas son innumerables, y en este caso fueron fundamentales siete: la organización, la disciplina, la creatividad, la confianza, la flexibilidad, y la capacidad autorreflexiva y analítica para el trabajo cooperativo. Pasar por este proceso de formación como docentes e investigadoras, también traslada al terreno de una experiencia más de libertad, porque forma capacidades, no solo profesionales, sino personales para ser y dejar ser, desafío que se asume día a día.
Referencias

Aristóteles. (1974). La política. Barcelona: Seix Barral.

Aristóteles. (2011). Ética a Nicómaco. Madrid: Tecnos.

Bárcena, F. (2005). La experiencia reflexiva en educación. Barcelona: Paidós.

Barragán, D. (2013). Cibercultura y prácticas de los profesores. Entre hermenéutica y educación. Bogotá: Universidad de La Salle.

Berge, A. (1959). La libertad en la educación. Buenos Aires: Kapelusz.

Berlin, I. (2004). Dos conceptos de libertad. Madrid: Alianza.

Bieri, P. (2001). El oficio de ser libre. Barcelona: Ariel.

Carr, W. (1999). Una teoría para la educación. Hacia una investigación educativa crítica. Madrid: Morata.

Elliott, J. (1990). La investigación-acción en educación. Madrid: Morata.

Fernández, M., y Betancourt, W. (2006). El concepto de la libertad de John Stuart Mill y su influencia ético-pedagógica en la formación del ciudadano de hoy. Bogotá: Universidad de La Salle.

Festugiére, A. (1953). La libertad en la Grecia antigua. Barcelona: Seix Barral.

Freire, P. (2011). La educación como práctica de la libertad. México: Siglo XXI.

García, M., y Sánchez, M. (1992). Alternativa participativa para educar en libertad. Bogotá: Pontificia Universidad Javeriana.

Gómez, M., y González, I. (1991). Incidencias de la práctica pedagógica y autoritaria en el ejercicio de la libertad y la autonomía de las alumnas de la concentración escolar San Agustín de Popayán. Bogotá: Pontificia Universidad Javeriana.

Hegel, G. (1985). Fenomenología del Espíritu. Madrid: Fondo de Cultura Económica.

Hovasse, C. (1973). Cómo educar a los niños en la libertad. Barcelona: Fontanella.

Kemmis y McTaggart. (1988). Cómo planificar la investigación acción. Barcelona: Laertes.

Kemmis, S. (2011). Researching educational praxis: spectator and participant perspectives. British Educational Research Journal. Publicado el 22 de agosto.

Luckmann, T. (1996). Teoría de la acción social. Barcelona: Paidós.

Luckmann, P., y Berger, T. L. (2001). La construcción social de la realidad. Barcelona: Amorrortu.

Lyupen, W. (1967). Fenomenología existencial. Buenos Aires: Ediciones Carlos Lohlé.

Maclntyre, A. (2001). Tras la virtud. Barcelona: Crítica.

Martínez, C., Mejía, G., Restrepo, I., Vergara, J., y Ripoll, K. (1996). El desarrollo de la autonomía, mirada a cuatro instituciones educativas. Bogotá: Pontificia Universidad Javeriana. 
Morales, S, (2012). La libertad: una práctica del bienestar. Una propuesta desde la educación física. Bogotá: Universidad Pedagógica Nacional.

Nancy, J. L. (1996). La experiencia de la libertad. Barcelona: Paidós.

Not, L. (1997). Las pedagogías del conocimiento. Bogotá: Fondo de la Cultura Económica.

Ortiz, S., y Díaz, D. (2008). El juego, entre la libertad y la escolarización. Bogotá: Universidad Pedagógica Nacional.

Patterson, O. (1993). La libertad en la construcción de la cultura occidental. Santiago de Chile: Editorial Andrés Bello.

Puig, J. M. (2001). La tarea de educar. Relatos sobre el día a día en una escuela. Madrid: Celeste Ediciones.

Puig, J. M. (2002). La educación moral en la enseñanza obligatoria. Barcelona: Horsori.

Puig, J. M. (2003). Prácticas morales. Una aproximación a la educación moral. Barcelona: Paidós.

Puig, J. M. (2010). Entre todos. Compartir la educación para la ciudadanía. Barcelona: Horsori Editorial, S. L.

Puig, J. M. (2011). La cultura moral como sistema de prácticas y mundo de valores. X Congreso Internacional de Teoría de la Educación. Barcelona: Universidad de Barcelona.

Puig, J. M. (2012). Cultura moral y educación. Barcelona: Editorial Graó.

Puig, J. M., Gijón Casares, M., Martín García, X., y Rubio Serrano, L. (2011). Aprendizaje-servicio y educación para la ciudadanía. Revista de Educación. Número extraordinario. Barcelona: Universidad de Barcelona, pp. 45-67.

Restrepo Botero, G. M., y Preciado Buitrago, J. C. (1999). Cartografía social. Tunja: Universidad Pedagógica y Tecnológica de Colombia.

Savater, F. (2004). Ética para Amador. Barcelona: Ariel.

Sánchez, O. (2013). Feminismo. Razón Pública. Bogotá: Razón Pública.

Schön, D. (1992). La transformación de profesionales reflexivos. Hacía un nuevo diseño de la enseñanza y el aprendizaje en las profesiones. Barcelona: Paidós.

Schütz, A. (1970). Fenomenología del mundo social, introducción a la sociología comprensiva. Buenos Aires: Paidós.

Sen, A. (2000). Desarrollo y libertad. Barcelona: Planeta.

Sennett, R. (2010). El Artesano. Barcelona: Anagrama.

Vásquez, F. (2013). El quehacer docente. Bogotá: Universidad de la Salle.

Yarce, J. (1974). La educación de la libertad. Medellín: Centro de Estudios Doctrinales. 


\section{Diálogo del conocimiento}

Comentario al artículo titulado: Libertad como fin educativo que transforma la práctica de un aula llanera, escrito por las profesoras Diana Yohana Gamboa Ladino y Yohanna Milena Nieto Gamboa. Experiencia desarrollada con estudiantes de primero de primaria, en la Institución Educativa Las Palmas, sede Brasilia (Villavicencio).

"Entre crayolas y experiencias de libertad", una frase evocadora de sueños que se van realizando a través de la experiencia reflexionada, de la posibilidad de construir conocimientos a partir de la práctica pedagógica de estas maestras, en quienes se ve florecer uno de los propósitos que como maestros de un país agobiado por la imposibilidad de ser y dejar ser, se nos presenta como fundamental: dar vía a una vida digna, desde la educación.

Una vida digna que apunta a fortalecer la construcción de argumentos en la relación con el otro y con lo otro, así mismo la toma de decisiones de manera autónoma y consensuada, favoreciendo la construcción de escenarios en los cuales se pueda hacer tangible "una vida juntos". Esto implica, necesariamente, comprender que el maestro hace parte del proceso y que desde los primeros años de escolaridad y de vida, se deben forjar "experiencias o prácticas de libertad", aparentemente tan sencillas, como permitir que los estudiantes vayan al baño durante las clases, sin pedir permiso al profesor. Pero que, como lo muestran las maestras, resultan trascendentales en el sentido de asumir responsabilidades frente a los procesos educativos propios y los del grupo en el que se está.

Esta complejidad de las experiencias de libertad, podría también ponerse en diálogo con los referentes culturales de las comunidades de los niños y en general del contexto del Municipio, el cual por tradición tiene una relación muy fuerte con el campo, con la ruralidad y, por ende, con otras maneras de interactuar con su medio y con todo lo que allí habita. Esto puede convertirse en otro de los hilos que maticen la trama de lo que implica formar en y para la libertad, además de ampliar el campo de acción del maestro, identificando otros actores sociales y circunstancias, que intervienen en los procesos educativos y en las mismas ideas de lo que significa ser libre o vivir dignamente.

En este sentido, se reitera la urgencia y pertinencia de miradas plurales frente al mundo, de "estar juntos" en el devenir de la vida y desde allí reconocerse, proponer formas de leer realidades, construirlas y transformarlas. En suma, se trata de interiorizar la "incertidumbre de vivir",no como obstáculo, sino como lo diría Freire: como "lo inédito viable", lo que aún no somos, pero podemos ser. Y ese podemos ser, pasa por el diálogo con otros, por el debate, reflexión y compromiso social, ético y político, que todos tenemos en el proceso de vivir juntos.

De ahí la relevancia de este trabajo, pues resalta el lugar de estas reflexiones desde acciones concretas con los niños, los cuales a veces son sub-estimados en el sentido de creer que ellos deben hacer lo que los adultos indiquen, pasando por alto su lugar de conocimiento $y$, por consiguiente, su responsabilidad en los procesos educativos propios y de quienes los rodean. Interesante planteamiento, aquel de fomentar experiencias de libertad, desde la posibilidad de "ser dueño de sí mismo, de dar lo mejor de sí, de trabajar con otros y de habitar con el mundo de manera propositiva"; esta es la invitación que nos deja la experiencia presentada a los maestros de un país que se quiere enrutar hacia el conocimiento de la diversidad biológica y cultural que habita en él, para desde allí generar escenarios que permitan la vida en todas sus manifestaciones.

Leidy Marcela Bravo 